\section{EAT: A PL/1 entropy analysis program}

\author{
HANS AIGNER \\ International Group Plans, Inc. \\ 2100 M Street, $N . W$., Washington, D.C. 20063
}

and

\section{R. DARCY \\ Department of Political Science \\ Oklahoma State University, Stillwater, Oklahoma 74074}

Entropy analysis has been shown to be an extremely flexible approach to the multivariate analysis of categorical data (Garner, 1962; Mathai \& Rathie, 1975; Theil, 1967; Watanabe, 1969). The EAT program performs an entropy analysis on up to 20 variables, some of which are defined as dependent and some of which are defined as independent. Depending on the options selected, the program will reduce the data to a weighted set of types (Darcy \& Aigner, 1976), calculate the entropy of each variable, calculate the distributional constraints present in a set of variables, calculate the internal constraints present if there is more than one dependent variable, and, for the set of dependent variables, calculate the explanatory power of each combination of the independent variables. The program permits extensive recoding of variables and accepts weighted data.

Program Parameters. Program parameters define the input data and specify program options desired. They consist of a series of key words followed by an equal sign $(=)$ and the appropriate parameter. They can be read in any order and are separated from one another by one or more blanks. The entire series of parameters is ended with a semicolon (;).

\section{Table 1}

Defining Variables

$\begin{array}{ll}\begin{array}{l}\text { VARIABLES }=n \text { or } \\ \text { V=n (required) }\end{array} & \begin{array}{l}\text { Specifies the number of variables ( } \mathrm{n} \text { ) to } \\ \text { be read in. Either form may be used. }\end{array} \\ \text { LOCATION(i)=j or } & \begin{array}{l}\text { Specifies the column location of the ith } \\ \text { variable to be } \mathrm{j} \text {. Either form may be used. }\end{array} \\ \text { CODES }(\mathrm{i})=\mathrm{j} \text { or } & \begin{array}{l}\text { Specifies the number of possible codes } \\ \text { for the ith variable, after recording, to be }\end{array} \\ \mathrm{j}(\mathrm{i})=\mathrm{j} \text { (required) } & \begin{array}{l}\text { opither form may be used. If the weight } \\ \text { option is used, specify } \mathrm{j} \text { to be } 1 \text { for the } \\ \text { weight variable. }\end{array}\end{array}$

NAME(i)="somename' Specifies somename as the name of the ith variable. Somename can be any set of special characters, numbers, blanks, or letters of up to 15 characters. The names need not be unique, but each must be specified.

\section{DEPENDENT $=\mathbf{n}$} (conditional)

INDEPENDENT $=\mathbf{n}$ (conditional)

\section{WEIGHT $=0$ or WEIGHT $=1$ \\ (conditional)}

WEIGHT $=n$ (conditional) (optional)
WIDTH=n

following the dependent variables are to be considered the independent variables.

Specifies that the last variable is to be treated as a weight factor (WEIGHT=1) or that the raw data are not weighted (WEIGHT=0). Default: WEIGHT $=0$.

Required if WEIGHT $=1$ is specified. $n$ is the total number of data cases being read in.

Specifies the width of the weight factor in $\mathbf{n}$ columns. If the weight factor is in more than one adjacent column, the LOCATION, or L, parameter is assumed to specify the beginning column of the variable. Default: WIDTH $=1$.

Table 2

Program Options (All Are Optional or Conditional)

$\begin{array}{ll}\text { ANALYSIS }=1 \text { or } & \text { Specifies independent-dependent vari- } \\ \text { ANALYSIS }=0 & \text { able entropy analysis is to be performed } \\ & \text { (ANALYSIS =1) or that it is not to be } \\ \text { performed (ANALYSIS }=0 \text { ). If ANALY- } & \text { SIS }=0 \text {, only the REDUCE portion of the } \\ & \text { program will execute. Defaul: ANALY- } \\ & \text { SIS }=0 .\end{array}$

PRINT $=0$ or PRINT $=1$

Specifies that reduced types will be printed (or output to terminal) (PRINT=1) or not printed (PRINT=0). Default: PRINT=0.

OUTPUT $=1$ or

OUTPUT $=0$

Specifies that the reduced types are to be output to some unit specified by the user (OUTPUT $=1$ ) or that they are not to be output (OUTPUT=0), Default: OUTPUT $=0$.

RECODE $=1$ or RECODE $=0$

Specifies that recoding is to be done $(\mathrm{RECODE}=1)$ or that it is not to be done (RECODE $=0$ ). Default: RECODE $=0$.

STOP $=1$ or $S T O P=0$ Specifies that input of data is to cease after a specified number of cases are read (STOP=1) or that data are to be read until all cases are read in (STOP=0). Default: $S T O P=0$.

STOPAT $=\mathbf{n}$

(conditional)

Required if $S T O P=1$ is specified. Specifies that only $n$ cases are to be input.

DIMENSION $=\mathrm{n} \quad$ Specifies space to be allocated for reduced data for independent-dependent variable entropy analysis. If DIMENSION $=0$, then $50000 /(5+$ INDEPEN DENT) will be the maximum number of types which can be analyzed. Specification of a larger $n$ raises this limit. Default (recommended): DIMENSION $=0$.

Recoding. Extensive recoding of data is permitted. Any recoding statements allowed within the $\mathrm{PL} / \mathrm{I}$ language may be used. This allows for DO loops, BEGIN blocks, IF statements-whatever is appropriate. The variable to be recoded is called $\operatorname{VAR}(\mathrm{n})$, where $\mathrm{n}$ represents the order in which the variable was read in. Variables are assumed to be characters. This allows 
numbers, letters, and special characters and symbols to be read in. Thus,

$$
\text { IF VAR(4) > ' } 5 \text { ' THEN } \operatorname{VAR}(4)=\text { '9'; }
$$

changes the value of the variable read in with $L(4)=n$ to 9 if it has a value larger than 5 . Each recode statement must end with a semicolon (;). As the data are character in form, single quotes (') must enclose the values specified. Any number of recode statements will be accepted. Cases may also be deleted from the data set. This is done by specifying DELETE $=1$; for such cases. Thus,

\section{IF VAR(8) $=$ ' 2 ' THEN DELETE $=1$;}

will cause all cases which have the eighth variable equal to 2 to be deleted.

Input Data. Data must reside on one logical record per case. The record may be up to 500 bytes (columns) long. Each variable must reside on one byte (column) only. The weight variable is an exception. It may reside on up to seven bytes. A maximum of 20 variables (including the weight) may be specified. These must reside on 20 or fewer bytes (columns) total. The data may include numbers, letters, and any special characters available. The weight variable, however, must consist of numbers.

Output. The entropy analysis has two parts: the description of the dependent variables and the explanatory power of the independent variables. First, the entropy and maximum entropy of each dependent variable along with the distributional and internal constraints of the dependent variables, taken together as a set, are printed. The proportion of the total possible variation (i.e., maximum entropy) of the set of dependent variables accounted for by the distributional and internal constraints are also presented. Next, the explanatory power of each possible combination of independent variables is examined. The program outputs the observed and maximum entropy for the dependent and independent variables taken together in every combination of independent variables. It also prints the entropy and maximum entropy of the various combinations of independent variables alone. Finally, the degree to which each combination of the independent variables can explain the distribution of the dependent variables is calculated and output.

Computer and Language. Versions of the program have been tested on an IBM 370/145 and an IBM 370/ 168 using the PL/1 optimizing compiler and on an IBM 360/50 using the PL/1 F compiler.

Availability. Copies of an EAT user's guide, a source listing of the program, and a source deck are available from Hans Aigner, International Group Plans, Inc., 2100 M Street, N.W., Washington, D.C. 20063.

\section{REFERENCES}

Darcy, R., \& Aigner, H. REDUCE: A program for reducing the number of cases in data sets and analyzing the constraints present within a series of variables. Journal of Marketing Research, 1976, 13, 410-411.

GARNER. W. Uncertainty and structure as psychological concepts. New York: Wiley, 1962.

Mathai, A. M., \& Rathie, P. N. Basic concepts in information theory and statistics. New York: Halsted. 1975.

THEIL, H. Economics and information theory. Chicago: Rand McNally, 1967.

WATANABE, S. Knowing and guessing. New York: Wiley. 1969.

(Received for publication April 21, 1977; revision accepted June 21.1977. ) 